\title{
Contenido de Fibra Dietaria de Inflorescencias de Palmas Procesadas
}

\author{
Dora Centurión-Hidalgo*, Judith Espinosa-Moreno, Efraín de la Cruz-Lázaro y \\ Erika Gómez-García \\ División Académica de Ciencias Agropecuarias, Univ. Juárez Autónoma de Tabasco, \\ Av. Universidad s/n, Zona de la Cultura, Col. Magisterial, C.P. 86000, Villahermosa, Centro, \\ Tabasco-México. (e-mail: dora-centurion@usa.net, juespinosa@hotmail.com, \\ elazaro@hotmail.com,gge99@hotmail.com) \\ * autor a quien se debe dirigir la correspondencia
}

Recibido Ago. 24, 2010; Aceptado Oct. 14, 2010; Versión Final recibida Nov. 18, 2010

\section{Resumen}

Se evaluó el efecto del procesamiento y de la madurez en el contenido de fibra dietaria de cuatro inflorescencias de consumo tradicional (Chamaedorea alternans, Chamaedorea tepejilote, Chamaedorea sp y Astrocaryum mexicanum). Se formaron tres grupos de acuerdo a la masa y se aplicaron tres tratamientos: crudo, asado y cocido. Las muestras tratadas se secaron y envasaron para determinar posteriormente el contenido de fibra dietaria. Se aplicó un diseño factorial con una distribución al azar $4 \times 3 \times 3$ (cuatro palmas, tres tratamientos y tres grupos). No se encontraron diferencias significativas al comparar el contenido de fibra dietaria total entre los tres grupos. El contenido de fibra dietaria soluble tuvo diferencias significativas $(p \leq 0.05)$ entre tratamientos siendo el tratamiento crudo el mejor. Se observó diferencias entre especies para fibra dietaria total y fibra dietaria insoluble.

\section{Dietary Fiber Content on Processed Palm Inflorescences}

\begin{abstract}
Processing effect and maturity stage on dietary fiber content of four traditionally consumed inflorescences (Chamaedorea alternans, Chamaedorea tepejilote, Chamaedorea $\mathrm{sp}$ and Astrocaryum mexicanum) were evaluated. Three groups were formed according to the mass and three treatments were applied: raw, roasted and boiled. Samples were dried and packed for dietary fiber determination. A randomly distributed factorial analysis $4 \times 3 \times 3$ (four palms, three treatments, three groups) was used. Total dietary fiber content was not significantly different $(p \leq 0.05)$ between groups. Soluble dietary fiber content was significantly different between treatments being the raw treatment the best one. It was observed that there were differences in total and insoluble dietary fiber contents between species.
\end{abstract}

Keywords: inflorescences, tropical palms, dietary fiber, maturity stage 


\section{INTRODUCCIÓN}

Las palmas se consideran como el segundo grupo vegetal en importancia económica, después de las gramíneas. En las regiones tropicales y subtropicales muchas comunidades dependen de ellas para su sustento. La contribución de estas plantas a la economía mundial, a los mercados y modos de vida local, es muy importante (Pérez y Rebollar, 2003). La variabilidad en formas de vida, la estructura de la comunidad de palmas en la selva y los múltiples usos de productos obtenidos de las palmas, está considerada como una fuente en los sistemas hortícolas y en el desarrollo sustentable (Mendoza y Oyama, 1999). En el caso de las palmas comestibles, destacan las inflorescencias masculinas tiernas de especies del género Chamaedorea (pacayas) y los frutos de algunas otras especies, como Bactris (Castillo et al., 1994). La guaya de joma, Chamaedorea tepejilote, se encuentra en América Central, donde es conocida como "pacaya", alrededor de la cual se ha desarrollado un comercio importante (Haynes y McLaughlin, 2000). Las inflorescencias, son una estructura que contiene las flores, que incluye brácteas y pedúnculos (Hodel, 1992).

La pacaya forma parte de la cultura alimentaria rural de Guatemala (Araya et al., 1981), el INCAP (Instituto de Nutrición de Centro América y Panamá) ha realizado estudios de su valor nutritivo, y encontraron que aporta proteína y minerales, principalmente calcio, a la dieta, mientras que IbarraManríquez et al. (1997) describieron una cultura del consumo de la inflorescencia de la especie Astrocaryum mexicanum en el entorno de Los Tuxtlas, Veracruz, México, donde se conoce como "chocho".

En particular, el World Conservation Monitoring Center (WCMC) y el United Nations Enviroment Programe (UNEP) han realizado estudios de mercadeo de la inflorescencia de palma Chamaedorea tepejilote, conocida como pacaya, tepejilote, guaya de joma o joma. La demanda de esta especie se presenta principalmente en el sureste de México y en algunos países de Centroamérica. En el caso de México, la demanda se localiza en los estados donde crece de manera natural: Chiapas, Oaxaca, Veracruz y Tabasco. En Guatemala y Honduras, la oferta sobre la comercialización la realizan dos compañías de conservas de tepejilote que abastecen los mercados de nostalgia en Estados Unidos y Canadá (Flores, 2002).

El alimento, por simple que sea, trae consigo una serie de tradiciones, costumbres y legados, como es el caso en la zona de la sierra del estado de Tabasco, donde las inflorescencias de las palmas como la escoba (Cryosophila stauracantha), Guacta (Bactris gracilior), guaya de cerro (Chamaedorea pinnatifrons), guaya de montaña (Chamaedorea sp), guayita de río (Chamaedorea cataractarum), pojay (Geonoma interrupta var. interrupta) y joma (Chamaedorea tepejilote) entre otras, son consumidas por la población católica durante la cuaresma (Centurión et al., 2003). En un estudio sobre la composición química proximal de las inflorescencias de Astrocaryum mexicanum, Chamaedorea altenans y Chamaedorea tepejilote se encontró un contenido de $24.92 \%$ de proteína, 1.62 a $2.26 \%$ de lípidos y de 9.84 a $12.76 \%$ de fibra cruda (CenturiónHidalgo et al., 2009). La fibra cruda está constituida por celulosa, lignina y pentosanas, que se forman junto con pequeñas cantidades de sustancias nitrogenadas de las estructuras celulares de los vegetales. La Asociación Oficial de Químicos Analíticos (AOAC), definió el término fibra bruta como la fracción que se pierde en la incineración del residuo seco obtenido tras la digestión de las muestras con $\mathrm{H}_{2} \mathrm{SO}_{4}$ al $1.25 \%$ y NaOH al $1.25 \%$ bajo condiciones específicas (Hart y Fisher, 1991). La fibra dietaria puede ser definida como el grupo complejo de sustancias de las plantas que son resistentes a las enzimas digestivas (Man, 1999). No hay duda del rol importante que se le adjudica a la fibra dietaria tanto en la prevención como en el tratamiento de patologías como diabetes, obesidad, constipación, arterioesclerosis, etc. De allí el interés en conocer el aporte de fibra dietaria de los alimentos y más aún su desglose en fibra soluble e insoluble dado que se le adjudican roles fisiológicos diferentes (Pak, 2002).

La fibra dietaria puede ser soluble (hidrosoluble) o insoluble, según sus componentes, en una solución acuosa. La fibra soluble comprende polisacáridos como las pectinas, gomas, mucílagos; todos presentan una alta capacidad de retención de agua y algunas de ellas son altamente viscosas en solución acuosa como la hemicelulosa, cutina, suberina. La fibra insoluble comprende a la celulosa, lignina, cutina, suberina, y la mayor parte de las hemicelulosas, que son 
componentes de la pared celular de los cereales y las verduras (Milke, 2002). La fibra insoluble es muy efectiva en aumentar el volumen fecal debido a que pasa intacta a través de tracto gastrointestinal y contribuye a la formación de heces voluminosas y suaves, disminuyendo así el tiempo de tránsito intestinal y la presión colónica. Por esta razón tiene poco efecto metabólico. La fibra soluble, especialmente cuando aumenta la viscosidad del contenido intestinal, tiene mayor efecto en reducir los niveles plasmáticos de colesterol, reducir la respuesta glicémica postprandial y retardar el vaciamiento gástrico (Witting de Penna, 2002).

Se han encontrado variaciones en el contenido de las diferentes fracciones de fibra dietaria en vegetales, las cuales se deben a la tecnología aplicada (Herranz et al. 1983). La cocción en agua produjo un incremento en el contenido de fibra neutra, fibra ácida y celulosa, mientras que la fritura produjo una disminución drástica de fibra neutra, fibra ácida, celulosa y lignina. Se han observaron diferentes efectos (Anderson y Clydesdale, 1980) ya que el tostado incrementó significativamente la lignina del salvado con tan sólo pequeños efectos en otros componentes de la fibra; mientras que el calentamiento en húmedo tiende a solubilizar y destruir las pectinas en el salvado, las zanahorias y los chícharos. Por otro lado, Alfonzo (2000) encontró que un mismo tipo de tratamiento térmico puede tener efectos diferentes en el contenido de fibra dietaria de los alimentos y señaló que la cocción promueve el rompimiento de sus componentes (celulosa, hemicelulosa, lignina, pectina, gomas), además de propiciar la interacción y enlace de estas sustancias con proteínas y lípidos, así como la generación de cambios cualitativos y/o cuantitativos sustanciales que varían la composición total de la fibra dietaria al comparar el alimento crudo con el cocido.

Por lo antes expuesto, el objetivo del presente trabajo fue evaluar el efecto del procesamiento y el estado de madurez en el contenido de fibra dietaria de inflorescencias de palmas.

\section{MATERIALES Y MÉTODOS}

Inflorescencias. Se utilizaron inflorescencias de cuatro especies de palmas: guaya de cerro (Chamaedorea alternans), guaya de calzón (Chamaeadora sp.), joma (Chamaedorea tepejilote) y chapaya (Astrocaryum mexicanum) que fueron adquiridas en los mercados municipales de Teapa y Tacotalpa, Tabasco, México, en manojos de 20 unidades para las tres especies de Chamaedorea y por unidad en el caso de Astrocaryum.

Selección de grupos. Se pesaron cada una de las inflorescencias de las cuatro especies utilizadas y con los datos obtenidos se formaron tres grupos de acuerdo al peso (g), para el caso de Chamaedorea sp, Chamaedorea tepejilote y Astrocaryum mexicanum, incluyendo en el Grupo I a las inflorescencias de menor peso (que se consideran como las menos maduras), las de peso medio formaron el Grupo II (madurez intermedia) y las de mayor peso en el Grupo III (más maduras). En cuanto a Chamaedorea alternans, se formaron tres grupos considerando el diámetro central de la inflorescencia, donde el grupo I tiene a las de menor diámetro, el grupo II fueron las de diámetro intermedio y el grupo III las de mayor diámetro.

Tratamientos. Los tratamientos aplicados a cada grupo fueron: 1) cocido en agua, 2) asado y 3) sin calor. En los tratamientos crudo y cocido, a las inflorescencias se les eliminó la parte no comestible (bráctea). Para el tratamiento cocido se calentó en agua por $10 \mathrm{~min}$ a $85^{\circ} \mathrm{C}$. El tratamiento asado se realizó con la bráctea (cubierta), metiéndolas en las brazas de acuerdo a la cultura de las familias en las áreas rurales donde se consumen. Después de aplicado el tratamiento, las inflorescencias se separaron y deshidrataron en un horno a $60^{\circ} \mathrm{C}$ por $24 \mathrm{~h}$, para posteriormente envasar en recipientes de plástico con tapa de rosca (De la Cruz, 2005). Los tratamientos aplicados a cada inflorescencia fueron: 1) cocido en agua, 2) asado y 3) sin calor. En los tratamientos crudo y cocido, a las inflorescencias se les eliminó la parte no comestible (bráctea). Para el tratamiento cocido se calentó en agua por $10 \mathrm{~min}$ a $85^{\circ} \mathrm{C}$. El tratamiento asado se realizó con la bráctea (cubierta) y en las brazas de acuerdo a la cultura de las familias en las áreas rurales de consumo. Después de aplicado el tratamiento, las inflorescencias separaron y deshidrataron en un horno a $60^{\circ} \mathrm{C}$ por $24 \mathrm{~h}$ y posteriormente se envasaron en recipientes de plástico con tapa de rosca (De la Cruz, 2005). 
Determinación de fibra dietaria total (FDT). Se realizó de acuerdo con el método 991.43 de la AOAC (1997). Para lo cual se preparó el buffer MES/TRIS, con pH 8.2 y temperatura de $24^{\circ} \mathrm{C}$, disolviendo $19.52 \mathrm{~g}$ de ácido 2(N-morfolino) etanosulfónico (MES) (Sigma M8250) y $14.2 \mathrm{~g}$ tris (hidroximetil) aminometano TRIS (Sigma T 1503) en 1.7I de agua desionizada. El ajuste de pH se realizó con soluciones de $\mathrm{NaOH}$ y/o $\mathrm{HCl}$. Se utilizaron crisoles para fibra ASTM 40-60 mm a peso constante y se pesaron $0.500 \mathrm{~g}$ de celite Sigma C8656, a los que se agregaron $10 \mathrm{ml}$ de etanol al $78 \%$ y se filtró la capa formada bajo vacío, las muestras se secaron en estufa a $130^{\circ} \mathrm{C}$ por $1.5 \mathrm{~h}$ y se realizó su pesaje.

Se pesó $1 \mathrm{~g}$ de muestra por triplicado y se transfirió a matraces Erlenmeyer de $250 \mathrm{ml}$, que se les adicionaron $40 \mathrm{ml}$ de solución buffer MES/TRIS pH 8.2 a $24^{\circ} \mathrm{C}$ a cada una de las muestras. Se agitó hasta homogenizar, evitando que quedara muestra en las paredes. Se calentaron con agitación a $95^{\circ} \mathrm{C}$ por $10 \mathrm{~min}$, se le añadieron $100 \mathrm{ml}$ de a-amilasa termoestable (Sigma No. A3306), para posteriormente tapar los matraces con papel aluminio y continuar con la agitación por 30 min a $95^{\circ} \mathrm{C}$. Se dejaron enfriar los matraces a temperatura ambiente (el gel formado en las paredes se removió con espátula y se lavó con $10 \mathrm{ml}$ de agua destilada). Para luego ajustar el pH a 7.5 con $\mathrm{HCl}$.

Se colocaron los matraces en un baño de agua a $60^{\circ} \mathrm{C}$ por $10 \mathrm{~min}$. Se adicionaron $100 \mathrm{ml}$ de proteasa (Sigma P3910, $0.025 \mathrm{~g}$ de proteasa más $0.5 \mathrm{ml}$ de buffer MES/TRIS) a cada matraz. Se taparon los matraces y se continúo calentando $60^{\circ} \mathrm{C}$ por $30 \mathrm{~min}$ y $60 \mathrm{rpm}$, se ajustó el pH a 4.5 con $\mathrm{HCl} 0.0561 \mathrm{~N}$ a $60^{\circ} \mathrm{C}$. Se adicionaron $300 \mathrm{ml}$ de amiloglucosidasa Sigma A9913, se taparon nuevamente los matraces con papel aluminio y se mantuvieron a $60^{\circ} \mathrm{C}$ por 30 min y $60 \mathrm{rpm}$. Se adicionó etanol al $95 \%$ y $60^{\circ} \mathrm{C}$ en relación $4: 1$ (aproximadamente $225 \mathrm{ml}$ de etanol) y se dejó en reposo durante $1 \mathrm{~h}$, se filtró en crisoles para fibra previamente pesados utilizando vacío. El residuo se lavó con dos volúmenes de $15 \mathrm{ml}$ de etanol al $78 \%$, dos volúmenes de $15 \mathrm{ml}$ de etanol al $95 \%$ y dos volúmenes de $15 \mathrm{ml}$ de acetona. Se secaron los crisoles con residuo a $130^{\circ} \mathrm{C}$ durante hora y media, se pesaron, colocaron en la mufla durante 5 horas a $550^{\circ} \mathrm{C}$ y se pesaron. Los cálculos se realizaron de la siguiente manera:

Peso del residuos $(\mathrm{R})=$ peso $($ crisol + celite + muestra $)-$ peso $($ crisol + celite $)$

Peso de cenizas $(C)=$ peso $($ crisol + celite + cenizas $)-$ peso $($ crisol + celite $)$

Se consideró el peso de los triplicados expresado en miligramos.

$\% \mathrm{FDT}=[(\mathrm{R}-\mathrm{C})(\mathrm{P}$ de muestra en $\mathrm{mg})-1]$ * 100

Los valores de fibra dietaria total (FDT), fibra dietaria soluble (FDS) y fibra dietaria insoluble (FDI) se expresaron en $\mathrm{g}$ en relación a $100 \mathrm{~g}^{-1}$ de peso seco. Las determinaciones se realizaron por triplicado calculándose la media y la desviación estándar. La fibra dietaria total se determinó por medio de la adición de la enzima proteasa, para lo cual se adicionaron $300 \mathrm{ml}$ de amiloglucosidasa, se taparon nuevamente los matraces con papel aluminio, se mantuvieron a $60^{\circ} \mathrm{C}$ por $30 \mathrm{~min}$ y $60 \mathrm{rpm}$. Se filtró al vacío utilizando crisoles previamente preparados y pesados. El residuo se consideró como fibra dietaria insoluble y se lavó dos veces con volúmenes de $15 \mathrm{ml}$ de etanol al $78 \%$, dos veces con volúmenes de $15 \mathrm{ml}$ de etanol al $95 \%$ y dos veces con volúmenes de $15 \mathrm{ml}$ de acetona. Se secaron los crisoles a $130^{\circ} \mathrm{C}$ durante 90 min y se pesaron. Se pasaron a la mufla durante 5 horas a $550^{\circ} \mathrm{C}$ y pesaron. Los cálculos se realizaron de la siguiente manera:

Peso de residuos $(R)=$ peso $($ crisol + celite + muestra $)-$ peso $($ crisol + celite $)$

Peso de cenizas $(C)=$ peso $($ crisol + celite + ceniza $)-$ peso $($ crisol + celite $)$

Se consideró el peso promedio de los triplicados expresado en mg

$\%$ de fibra dietética insoluble $(\mathrm{FDI})=[(\mathrm{R}-\mathrm{C})($ peso de muestra en $\mathrm{mg})-1]$ * 100 
La fibra dietaria soluble se determinó por diferencia:

$\%$ FDS $=\%$ FDT $-\%$ FDI

Diseño de experimentos y análisis estadístico. Se utilizó un diseño estadístico factorial $4 \times 3 \times 3$ con distribución al azar (cuatro palmas, tres tratamientos y tres grupos). Para determinar diferencias entre tratamientos se realizó una prueba de comparación de medias de Tukey.

\section{RESULTADOS Y DISCUSIÓN}

No se encontraron diferencias significativas al comparar el contenido (porcentaje) de Fibra Dietaria Total (FDT) entre los grupos, aunque el contenido de Fibra Dietaria Insoluble (FDI) fue mayor en el Grupo II contenido que es diferente al que tuvieron los otros dos grupos. Por otro lado, el Grupo III presentó el mayor contenido de Fibra Dietaria Soluble (FDS), que es diferente al contenido que tuvieron los otros grupos. (Tabla 1).

Tabla 1: Comparación de medias (prueba de Tukey) de fibra dietaria entre grupos $(p=0.05)$. Medias con la misma letras dentro de grupos, son iguales estadísticamente $(p=0.05)$

\begin{tabular}{|c|c|c|c|}
\hline Grupo & $\begin{array}{c}\text { Fibra Dietaria Total } \\
(\text { FDT } \%\end{array}$ & $\begin{array}{c}\text { Fibra Dietaria Insoluble } \\
\text { (FDI) } \%\end{array}$ & $\begin{array}{c}\text { Fibra Dietaria Soluble } \\
\text { (FDS) } \%\end{array}$ \\
\hline I & $56.17 a$ & $51.94 a b$ & $4.24 \mathrm{~b}$ \\
\hline II & $57.76 a$ & $53.71 \mathrm{a}$ & $3.88 \mathrm{~b}$ \\
\hline III & $56.17 \mathrm{a}$ & $48.62 \mathrm{~b}$ & $7.76 \mathrm{a}$ \\
\hline
\end{tabular}

Es importante hacer notar que las inflorescencias de mayor peso (Grupo III) presentaron los mayores contenido de FDS, a pesar de que no presentaron diferencias significativas en la FDT, por lo que el consumirlas ayudaría a reducir los niveles plasmáticos de colesterol (Wittig de Penna, 2002). Al respecto, Valencia y Román (2004) recomiendan que, de la fibra que se consume, al menos el $30 \%$ deba ser fibra soluble; las inflorescencias estudiadas en este trabajo presentan esta proporción lo que indica la importancia de su consumo por las comunidades rurales.

En la Tabla 2 se observan diferencias significativas en el contenido de fibra de las cuatro inflorescencias. El mayor porcentaje de FDT y FDI se encontró en Astrocaryum mexicanum, valores que son diferentes estadísticamente a los que se obtuvieron en Chamaedorea tepejilote pero iguales estadísticamente a los encontrados en Chamaedorea sp y C. alternans. Con respecto a la FDS, el contenido fue estadísticamente similar para tres de las cuatro especies de palmas, siendo diferente estadísticamente Chamaedorea alternans que tuvo el menor contenido de FDS. Los contenidos de FDT de las inflorescencias de palmas evaluadas se encuentran dentro de los valores promedio de FDT para vegetales, que van del 45 al $72 \%$ (Gelroth y Ranhorta, 2001). Por lo que las inflorescencias de las palmas evaluadas representan una excelente fuente de fibra para los habitantes de la región donde se consumen.

Tabla 2: Comparación de medias (Prueba de Tukey) del porcentaje de fibra dietaria entre especies $(p=0.05)$. Medias con la misma letras son iguales $(p=0.05)$

\begin{tabular}{|l|c|c|c|}
\hline \multicolumn{1}{|c|}{ Especie } & $\begin{array}{c}\text { Fibra Dietaria Total } \\
(\text { FDT) } \%\end{array}$ & $\begin{array}{c}\text { Fibra Dietaria } \\
\text { Insoluble (FDI) } \%\end{array}$ & $\begin{array}{c}\text { Fibra Dietaria Soluble } \\
\text { (FDS) \% }\end{array}$ \\
\hline Chamaedorea sp & $48.71 \mathrm{c}$ & $42.64 \mathrm{c}$ & $6.06 \mathrm{a}$ \\
\hline Chamaedorea alternans & $47.63 \mathrm{c}$ & $45.43 \mathrm{bc}$ & $2.20 \mathrm{~b}$ \\
\hline Chamaedorea tepejilote & $54.73 \mathrm{~b}$ & $50.41 \mathrm{~b}$ & $4.34 \mathrm{ab}$ \\
\hline $\begin{array}{l}\text { Astrocaryum } \\
\text { mexicanum }\end{array}$ & $73.82 \mathrm{a}$ & $67.01 \mathrm{a}$ & $6.74 \mathrm{a}$ \\
\hline
\end{tabular}


Es importante hacer mención que no se han encontrado datos de fibra dietaria en la literatura consultada para ninguna de las tres especies de Chamaedorea estudiadas en este trabajo; sin embargo, Morón et al. (1997) reportaron que los valores de fibra dietaria son por lo general de 3 a 5 veces mayores que los valores de fibra cruda. En este contexto, otros investigadores han reportado valores del $2 \%$ de fibra cruda en Chamaeadora tepejilote (Castillo et al., 1994) y del $8 \%$ en Chamaeadora sp (Atchley, 1984) lo que puede convertirse hasta en un $40 \%$ de FDT. Los valores encontrados en este trabajo son mayores a los reportados por dichos autores, aunque en dichos trabajos no mencionan el método de la determinación para realizar los análisis ni las variables que tomaron en cuenta, tales como desarrollo o estado de madurez de la muestra. En el caso de A. mexicanum, De la Cruz (2005) desarrolló un producto deshidratando, moliendo y tamizando las inflorescencias, encontrando un $10.6 \%$ de fibra cruda (aproximadamente $50 \%$ de FDT), valores que se encuentran cercanos a los encontrados en el presente trabajo.

En la comparación de medias entre tratamientos (Tabla 3), no se encontraron diferencias para las variables FDT y FDI. Sin embargo, en el tratamiento crudo la FDS fue diferente estadísticamente de los tratamientos cocido y asado, pues mostró el mayor contenido y observando que el tratamiento térmico de procesado redujo significativamente el contenido de FDS. Al respecto, Ramulu y Udayasekhara Rao (1997) encontraron que el efecto del procesamiento no es uniforme en todos los alimentos Por ejemplo, el procesado de cereales (excepto el mijo) en chapati no tiene efecto en su contenido de fibra dietaria total e insoluble, pero el cocimiento a presión de legumbres resultó en un aumento significativo en el contenido de su fibra dietaria total después del procesado que se debió principalmente al aumento del contenido de fibra dietaria insoluble.

Tabla 3. Comparación de medias (Prueba de Tukey) entre tratamiento de procesamiento $(p=0.05)$. Medias con la misma letras son iguales $(p=0.05)$

\begin{tabular}{|c|c|c|c|}
\hline Tratamiento & $\begin{array}{c}\text { Fibra Dietaria Total } \\
\text { (FDT) }\end{array}$ & $\begin{array}{c}\text { Fibra Dietaria Insoluble } \\
\text { (FDI) }\end{array}$ & $\begin{array}{c}\text { Fibra Dietaria Soluble } \\
\text { (FDS) }\end{array}$ \\
\hline Crudo & $57.01 \mathrm{a}$ & $50.22 \mathrm{a}$ & $7.14 \mathrm{a}$ \\
\hline Asado & $55.82 \mathrm{a}$ & $51.62 \mathrm{a}$ & $4.01 \mathrm{~b}$ \\
\hline Cocido & $57.48 \mathrm{a}$ & $53.24 \mathrm{a}$ & $4.05 \mathrm{~b}$ \\
\hline
\end{tabular}

Después de aplicado el procesado térmico, se encontró que la FDT no presentó diferencias significativas, es decir que el calor no afectó el contenido de FDT. Por otro lado, los tratamientos no afectaron el contenido de FDI de los diferentes tipos de palmas, aunque los tratamientos térmicos (asado y cocido) disminuyeron el contenido de FDS. Se ha reportado que el tratamiento térmico afecta el contenido de FDS y FDI en leguminosas, donde se ha observado un aumento de aproximadamente el doble en el contenido de FDS (Alfonzo, 2000). Sin embargo, en las inflorescencias de palmas ocurrió lo contrario presentándose una pérdida de la FDS durante el proceso térmico. Por otro lado, Azizah y Zainon (1997) reportaron el efecto de los tratamientos térmicos de ebullición y asado en leguminosas y cereales, encontrando que la FDS disminuyó en el caso del trigo (Tritium vulgare), arroz (Oryza sativa) y soya (Glycine hispida), teniendo un comportamiento similar a las inflorescencias de palmas estudiadas.

La naturaleza soluble e insoluble de las fibras dietarias comprende diferencias en su funcionalidad tecnológica y efectos fisiológicos. Las fibras solubles están caracterizadas por su capacidad para aumentar la viscosidad y para reducir la respuesta glicémica y el colestrol plásmático. Las fibras insolubles están caracterizadas por su porosidad, su baja densidad y por su habilidad para aumentar el bolo fecal y disminuir el tránsito intestinal (Elleuch et al., 2010). Se ha estudiado el efecto del cocinado de dos métodos, uno asado convencional y el otro a presión en legumbres, hojas de vegetales, raíces y tubérculos de la fibra dietaria total y la fibra soluble e insoluble y se reportó un incremento significativo en la fracción de la fibra soluble y decremento en la fibra insoluble (Farhath Khanum et al., 2000). 


\section{CONCLUSIONES}

El contenido de fibra dietaria es diferente en cada una de las especies de palma estudiadas. El tamaño de las inflorescencias tuvo influencia en los contenidos de FDI y FDS de cada una de las palmas estudiadas. Se encontró que los tratamientos con la aplicación de calor (asado y cocido) no afectaron el contenido de FDT, mientras que el contenido de FDS fue afectado por los tratamientos con aplicación de calor.

\section{AGRADECIMIENTOS}

El presente trabajo se realizó como parte del proyecto de investigación "Rescate y conservación de germoplasma vegetal en riesgo de erradicarse en la sierra del estado de Tabasco" apoyado por Fondos Mixtos CONACyT-Tabasco con clave TAB-2003-03-11386.

\section{REFERENCIAS}

Alfonzo, G. G. C. Efecto del tratamiento térmico sobre el contenido de fibra dietética total, soluble $e$ insoluble en algunas leguminosas. Archivos Latinoamericanos de Nutrición 50 (3):281-285 (2000).

Anderson, N. E. and Clydesdale, F. M. Effects of processing on the dietary fiber contain of wheat bran, pureed green beans, and carrots. J. Food Sci. 45:1533-1537 (1980).

AOAC. Official Methods of Analysis of AOAC International. 17th Ed. The Association of Official Analytical Chemists. Maryland, USA (1997).

Araya, H., Flores, M. and Arroyave, G. Nutritive value of basic foods and common dishes of the Guatemala rural populations. Ecology and Food and Nutrition 11:174-175 (1981).

Atchley, A. A. Nutritive value of palms. Principes 28(3): 138-143 (1984).

Azizah, A. H., Zainon, H. Effect of processing on dietary fiber contents of selected legumes and cereals. Mal J Nutr 3: 131-137 (1997).

Castillo, M. J. J., Gallardo, N G. and Johnson, D. V. The pacaya palm (Chamaedorea tepejilote, Arecaceae) and its food use in Guatemala. Economic Botany 48 (1): 68-75 (1994).

Centurión, H. D., Espinosa, M. J., Poot, M. J. E., Cázares, C, J. G. Cultura alimentaria de la región Sierra de Tabasco. Editorial UJAT Colección José Ma. Pino Suárez. pp: 48-49 (2003).

Centurión-Hidalgo, D., Alor-Chávez, M. J., Espinosa-Moreno, J., Gómez-García, E., Solano, M. L., Poot-Matu, J. E. Contenido nutricional de inflorescencias de palmas en la sierra del estado de Tabasco. Universidad y Ciencia 25(3):193-199 (2009).

De la Cruz, C. A. A. Alternativa de un alimento no convencional propio de la región. Revista Salud Pública y Nutrición, Edición Especial No. 10-2005 Consultado en la Web el 15/05/2007 en la página electrónica: http:/www.respyn.uanl.mx/especiales/2005/ee-102005/documentos/estudiantesdoc.htm (2005).

Elleuch, M., Bedigian, D., Roiseux, O., Besbes, S., Blecker, C., Attia, H. Dietary fibre and fibre-rich by-products of food processing: Characterization, technological functionality and commercial applications: A review. Food Chemistry 124(2011): 411-421 (2010).

Farhath Khanum, Siddalinga Swamy, M., Sudarshana Krishna, K. R., Santhanam, K,. Viswanathan, K. R. Dietary fiber content of commonly fresh and cooked vegetables consumed in India. Plant Foods for Human Nutrition 55: 207-218 (2000). 
Flores, J. C. Tepejilote (Chamaedorea tepejilote). Informe de Mercadeo. World Conservation Monotoring Center (WCMC) y United Notions Enviroment Programe (UNEP) (2002).

Gelroth, J., Ranhorta, S. G. Food uses of fiber. En: Sungsoo Cho, S. (Ed.). Handbook of Dietary Fiber. Mark L. Dheher, New York. pp: 435-449 (2001)

Hart, F. L., y Fisher, H. J. Análisis Moderno de los Alimentos. Editorial Acribia, España. pp: 44-45 (1991).

Haynes, J. and McLaughlin, J. Edible palms and their uses. Fact sheet MDCE-00- 50-1. Extension. Institute of Food and Agricultural Sciences, University of Florida. pp: 4-6 (2000).

Herranz, J., Vidal-Valverde, C. y Rojas-Hidalgo, E. Cellulose, hemicellulose and lignin content of raw and cooked processed vegetables. J. Food Sci., 48: 274-275 (1983).

Hodel, D.R. Chamaedorea palms: the species and their cultivation. Internacional Palm Society, Allen Press, Lawrence, Kansas (1992).

Ibarra-Manríquez, G., Ricker, M., Angeles, G., Sinaca, C. S., Sinaca, C. M. A. Useful plants of Los Tuxtlas rain forest (Veracruz, México). Economic Botany 51: 363-376 (1997).

Man, M. J. de. Principles of Food Chemistry. T ${ }^{\text {rd }}$ Edition. An Aspen Publication, Maryland. 203 p. (1999).

Mendoza, A. and Oyama, K. Ecology management and conservation of potentially ornamental palms. Proc. of the $2^{\text {nd }}$ Inter Symp. on Ornamental Palms and other monocot forms tropics. Acta Hort. 486: 79-86 (1999).

Milke, P. Fibra dietética y funciones gastrointestinales. In: García, O.P.G. (Ed.). Enciclopedia de la fibra. Kellogg's de México, Querétaro, Qro. pp: 64-65 (2002).

Morón, C., Zacarías, I., Pablo, S. (Editores). Producción y manejo de datos de composición química de alimentos en nutrición. FAO. Dirección de Alimentación y Nutrición. Oficina Regional para América Latina y el Caribe. INTA. Universidad de Chile. Santiago de Chile. pp. 177-187. (1997).

Pak, D. N. Fibra dietética en verduras cultivadas en Chile. Archivos Latinoamericanos de Nutrición 50(1): 5-10 (2002).

Pérez, M y Rebollar, S. Anatomía y usos de las hojas maduras de tres especies de Sabal (Arecaceae) de la Península de Yucatán, México. Rev. Biol. Trop. 51(2):333-344 (2003).

Ramulu, R., Udayasekhara Rao, R. Effect of processing on dietary fiber content of cereals and pulses. Plant Foods for Human Nutrition 50: 249-257 (1997).

Valencia, G. F. E. y Román, M. M.O. La fibra como alimento funcional. VITAE, Revista de la Facultad de Química Farmacéutica 11(2): 12-17 (2004).

Witting de Penna, E., Serrano, L., Bunger, A., Soto, D., López, L., Hernández, N., Ruales, J. Optimización de una formulación de espaguetis enriquecidos con fibra dietética y micronutrientes para el adulto mayor. Archivos Latinoamericanos de Nutrición 52(1): 91-100 (2002). 